\title{
Organ Asymmetries as Correlates of Other Anomalies
}

\author{
Mason Barr, Jr.* \\ Departments of Pediatrics, Pathology, and Obstetrics, University of Michigan, Ann Arbor, Michigan
}

\begin{abstract}
Fetuses with deviations in the normal left versus right weight relationships of the paired viscera were examined for correlation with particular malformations, syndromes, or other common factors. Alterations of the normal pattern were observed among cases with cytogenetic anomalies, diaphragmatic hernia, renal duplication, dyshydramnios, and single umbilical artery. A reversal of the left versus right weight pattern was found in the adrenals of cases with hepatic situs inversus but not in those with heterotaxic cardiac lesions. Recipient twins in the twin-twin transfusion syndrome had reduced left lung weights, presumably because of inhibition of lung growth by the enlarged heart. The side of umbilical artery absence did not predict either the nature or complexity of associated malformations. However, it is suggestive that there is discordance between the side of the missing artery and unilateral renal abnormality and that there is preferential absence of the right or left artery, depending on the karyotype.

๑) 2001 Wiley-Liss, Inc.
\end{abstract}

KEY WORDS: adrenals; kidneys; lungs; umbilical arteries; asymmetry; anomalies

Humans, as all vertebrates, are classified as bilaterally symmetrical animals. However, it is commonly recognized that considerable asymmetry exists in the normal individual. There is obvious asymmetry in the heart, lungs, and liver and in the position of the abdominal viscera. Several of the viscera are paired but normally show a measure of weight asymmetry. These include the lungs, kidneys, adrenals, and gonads. In this article, consideration will be given to deviations from the usual left-right weight relationship of the

*Correspondence to: Mason Barr, Jr., M.D., Pediatric GeneticsTeratology, 1924 Taubman Center, Box 0318, University of Michigan Hospitals, Ann Arbor, MI 48109.

E-mail: barr@med.umich.edu

Received 8 November 2000; Accepted 1 December 2000 paired viscera (except the gonads) and their correlation with certain anomalies or disorders. This study will not address the question of total organ weight deviation, only the deviation from the normal difference of weight between organs of opposite position. Attention will also be given to the morphologic associations of single umbilical artery, depending on the side of arterial absence.

\section{MATERIALS AND METHODS}

The study group for this investigation was drawn from the University of Michigan Teratology Unit data set, a series of 4,347 fetal autopsies performed in consistent fashion by one prosector. Cases with more than minimal maceration, death more than 12 hours after delivery, body weight less than $50 \mathrm{~g}$, or prior formalin fixation were excluded. Not all cases had complete sets of weights of the organs under consideration. Among the 2,062 cases for analysis, there were 2,033 for adrenals, 1,954 for kidneys, and 2,024 for lungs.

The methodology of collecting fetal visceral weight data and calculating normal growth curves for human fetuses has been published [Barr et al., 1994]. Using only normal fetuses, growth curves were computed for left versus right organ for each of the paired viscera: adrenals, kidneys, and lungs (Fig. 1). Although the combined (i.e., left plus right) weight of these organs plotted against body weight is best described by power equations with polynomial exponents [Barr et al., 1994], the relation of left versus right organ is adequately described in each case by a linear equation, indicating no preferential acceleration or deceleration of growth on either side. From the regressions and their standard deviations, z-scores for left versus right were calculated for each set of paired viscera in the study group. The formulae used, with organ weights in grams, were

$$
\begin{aligned}
& \text { zLvR adrenal: }(\mathrm{L} \text { adrenal }-(-0.01522 \\
& \quad+(1.14133 \times \mathrm{R} \text { adrenal })) /(0.08953 \times \mathrm{R} \text { adrenal }) \\
& \text { zLvR kidney: }(\mathrm{L} \text { kidney }-(0.00228 \\
& \quad+(1.02150 \times \mathrm{R} \text { kidney }))) /(0.07566 \times \mathrm{R} \text { kidney }) \\
& \text { zLvR lung: }(\mathrm{L} \text { lung }-(0.09009 \\
& \quad+(0.79678 \times \mathrm{R} \text { lung }))) /(0.04315 \times \mathrm{R} \text { lung })
\end{aligned}
$$



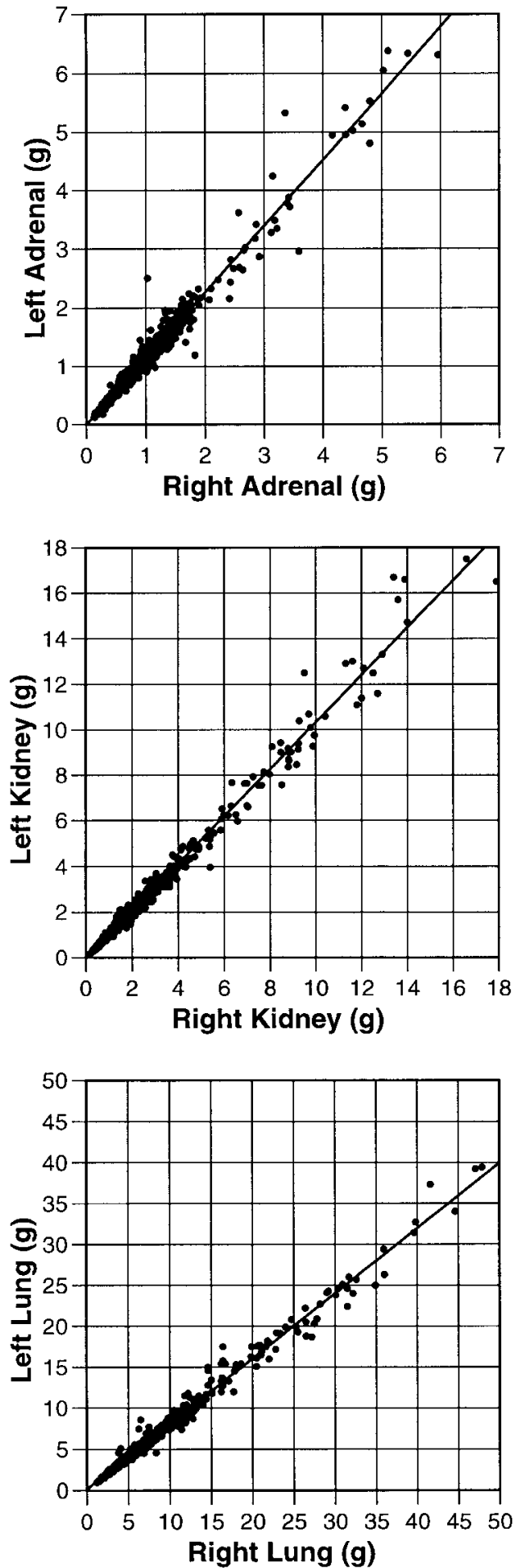

Fig. 1. Scatterplots of weight of left versus right adrenal, kidney, and lung. Note the inequality of the adrenals and of the lungs that remains constant throughout gestation, whereas renal weights remain nearly equal to each other.

Thus, on average the right adrenal accounts for $47 \%$ of total adrenal weight, the right kidney for $49.5 \%$ of total renal weight, and the right lung for $56 \%$ of total lung weight. This type of analysis does not inform about total organ weight, nor does it necessarily distinguish between one side being too heavy and the opposite side being too light. However, from prior studies that distinction can often be made [Barr, 1994, 1997, 1998].

When the total population studied $(2,062)$ was divided into morphologically normal (920) or abnormal $(1,142)$ fetuses, the distributions of left versus right organ z-scores for the normal fetuses formed the expected normal bell-shaped figures. However, in the case of the abnormal fetuses, the ends of the curves were considerably extended, reflecting the presence of many aberrations. The reports of cases with z-scores outside the $95 \%$ prediction interval were screened for malformations, diagnoses, and other common factors. If a particular factor appeared to occur with unusual frequency, the distribution of z-scores for all cases with that factor was examined in more detail. Many diagnoses in the study group were represented by too few cases to perform a meaningful analysis of z-score distribution.

\section{RESULTS \\ Paired Viscera}

Among fetuses with one of the common autosomal trisomies, deviations from the usual left-right relationship were seen (Fig. 2). In trisomy 21 (108), there was a shift toward more equal weight of the adrenal glands from the normal pattern of the left gland being the heavier. The left lung is normally lighter than the right lung, but this difference between the two sides was more pronounced in fetuses with Down syndrome. The lung $\mathrm{z}$-score distributions were unaffected by the presence or absence of major cardiac defects. No deviation from the usual pattern was found for the kidneys. In trisomy 18 (39), the predominant feature seen in the three organs was an excessive number of outliers, both high and low, when compared to the normal left-right weight patterns, although there also appeared to be a trend to more equal adrenal weights. In trisomy 13 (35), there was a tendency for the left kidney to be lighter (or the right, heavier), and the left lung to be heavier (or the right, lighter). The adrenals showed the normal pattern. In fetuses with Turner syndrome (35), no significant deviations from the expected left-right weight differential of the three organs were found (data not shown).

The distributions of z-scores of left versus right lung weight were examined for fetuses associated with oligohydramnios (311) or polyhydramnios (131). In both instances, there were excessive numbers of outliers, both high and low, reflecting the wide variety of structural abnormalities associated with abnormal amniotic fluid volume (Table I).

In anencephaly (92), the adrenals showed an excess of instances in which the glands were more nearly equal in weight than in normal fetuses (Table I). However, this may be misleading because the adrenals in anencephaly are typically diminutive, and small weight differences may be overinterpreted. For fetuses with myeloschisis (70), there were no appreciable 

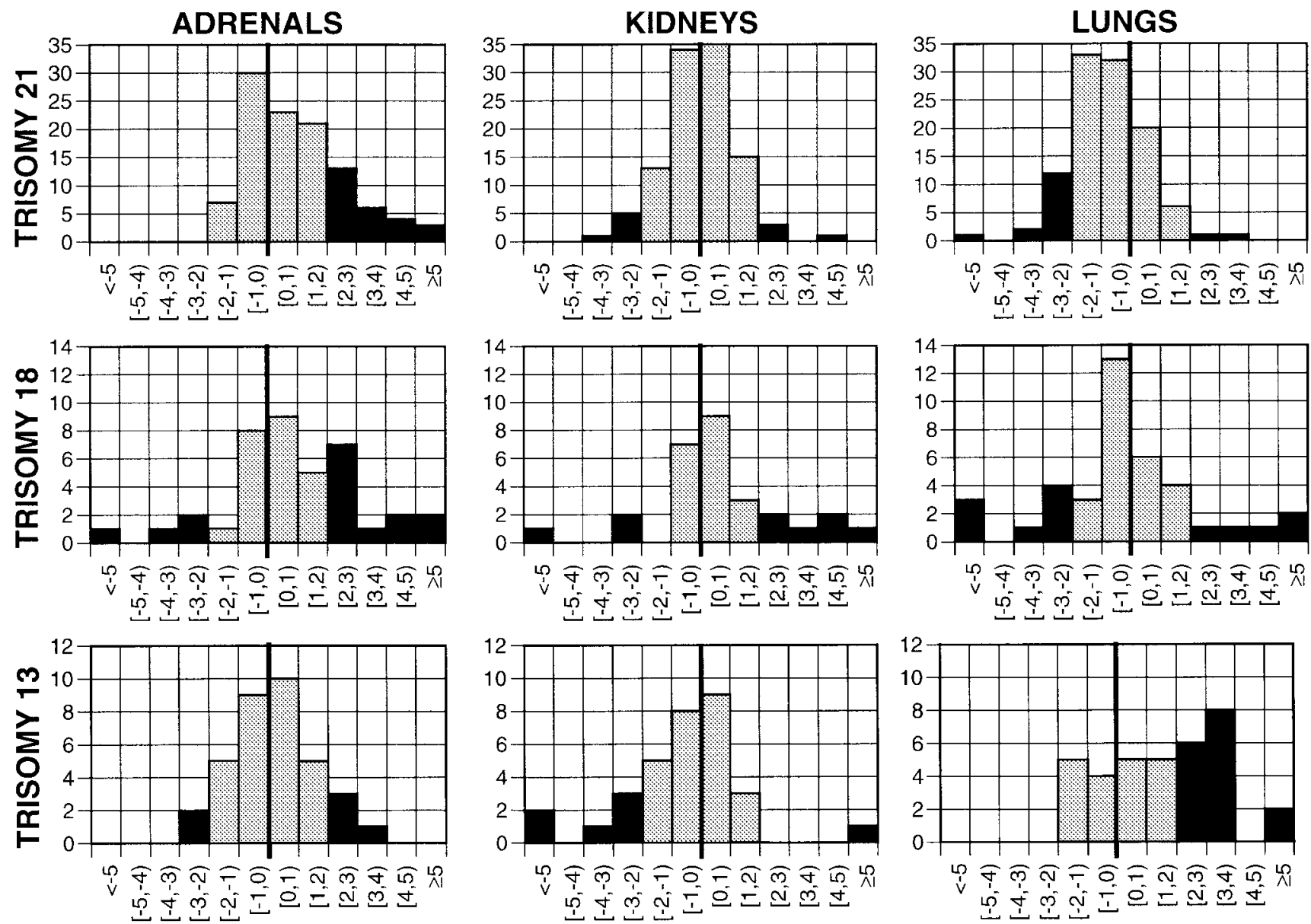

Fig. 2. Z-score distributions of left versus right adrenals, kidneys, and lungs for trisomies 21,18 , and 13 . The shaded area is \pm 2 SD (approximately the $95 \%$ prediction interval). The black areas are those cases falling outside the $95 \%$ prediction interval. A heavy bar marks the central point $(\mathrm{Z}=0)$.

deviations from the usual weight patterns of the three organs studied (data not shown).

The distribution of z-scores of left versus right kidney weight in instances of unilateral renal duplication was examined. As expected, the duplicated kidney was heavier, whether on the left (21) or the right (15) (Fig. 3 ). It is possible that some cases of "fused renal duplication" were not recognized at autopsy and account for other cases in which the left-right weights deviated from the usual pattern of near equality.

In case of diaphragmatic hernia (33 left, 10 right), the lung weights were most reduced on the side of the herniation, the exceptions being found in cases with very complex malformation patterns or the presence of pulmonary sequestration in the contralateral lung (Table I).

An interesting observation was made in cases affected by the twin-twin transfusion syndrome. The recipient twins (38) were found to have reduced weight of the left lung, an alteration not found in the donor twins (28) (Fig. 3). It has been previously reported that one of the earliest manifestations of hemodynamic imbalance in the twin-twin transfusion syndrome is cardiac enlargement caused by myocardial hyperplasia in the recipient twin [Pridjian et al., 1991]. The observed lung weight discrepancy is likely a mechanical effect on the developing lung from the enlarged heart.

TABLE I. Left Versus Right Z-Score Distributions for Selected Conditions and Organs

\begin{tabular}{llccc}
\hline & & \multicolumn{3}{c}{ Z-score } \\
\cline { 3 - 5 } Disorder & Organ & $\begin{array}{c}<-1.95 \\
(2.5 \%)\end{array}$ & $\begin{array}{c}-1.95 \text { to }+1.95 \\
(95 \%)\end{array}$ & $\begin{array}{c}>+1.95 \\
(2.5 \%)\end{array}$ \\
\hline Polyhydramnios & Lungs & 28 & 81 & 21 \\
Oligohydramnios & Lungs & 38 & 231 & 41 \\
Anencephaly & Adrenals & 7 & 55 & 27 \\
Diaphragm defect left & Lungs & 21 & 6 & 5 \\
Diaphragm defect right & Lungs & 1 & 0 & 9 \\
\hline
\end{tabular}


Left Renal Duplication

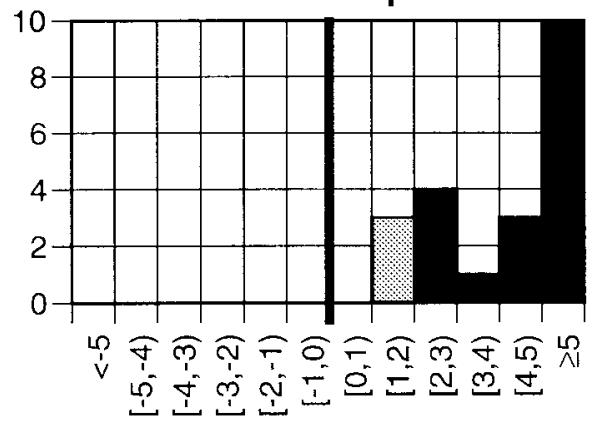

Recipient Twin

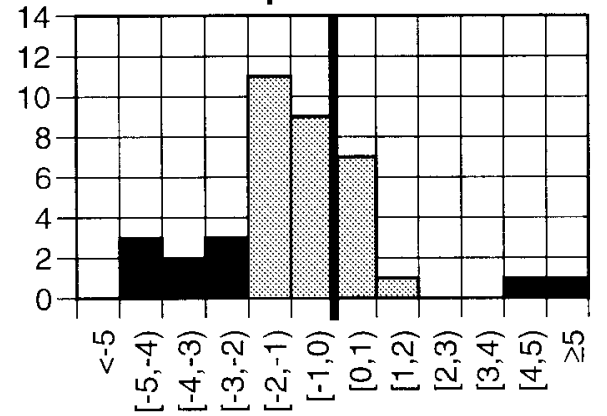

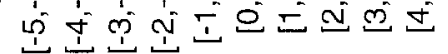

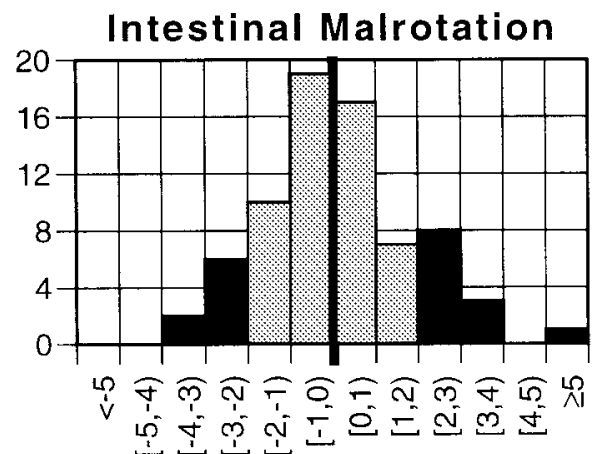

Right Renal Duplication
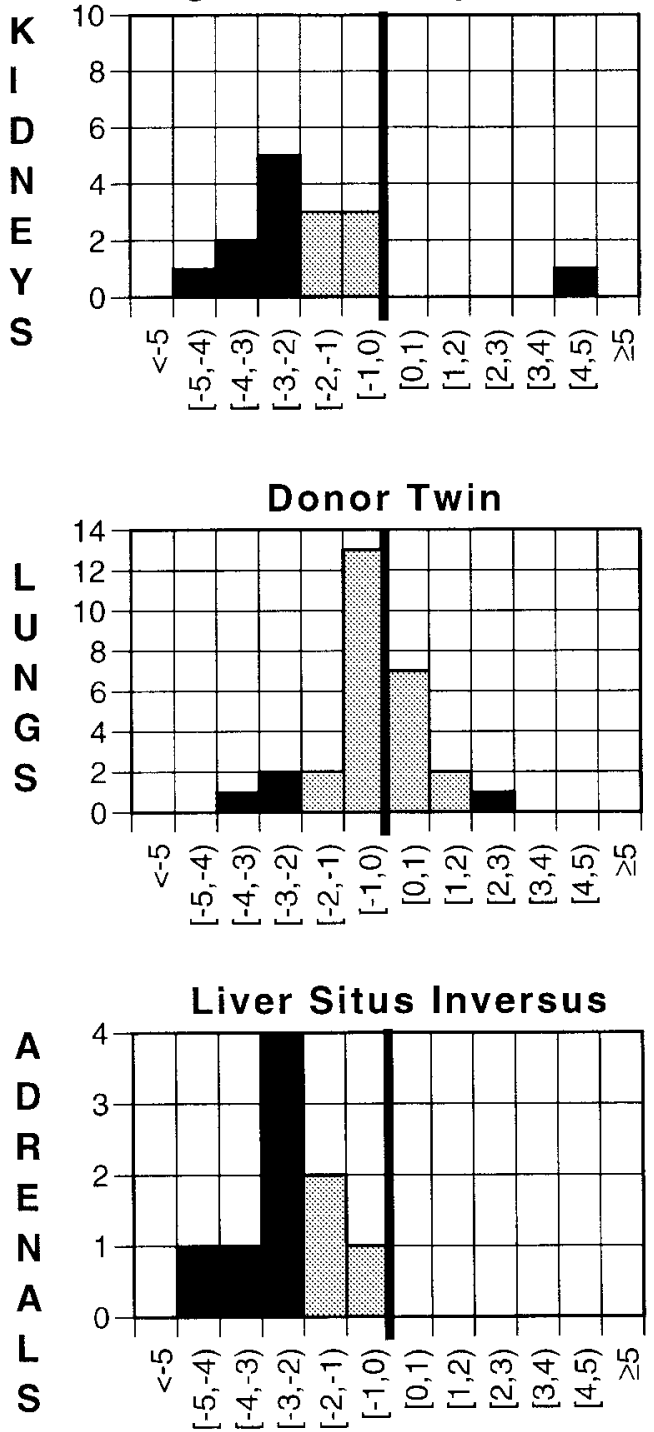

Fig. 3. Z-score distributions of left versus right for kidneys in unilateral renal duplication, lungs in the twin-twin transfusion syndrome, and adrenals in intestinal malrotation compared to hepatic situs inversus. The shaded area is \pm 2 SD (approximately the $95 \%$ prediction interval). The black areas are those cases falling outside the $95 \%$ prediction interval. A heavy bar marks the central point $(\mathrm{Z}=0)$.

Cases with situs ambiguity were examined. When situs inversus of the liver was used as the selection criterion (9), left-versus-right adrenal weights were found to be reversed from the usual pattern (Fig. 3). No such effect was found in cases with intestinal malrotation (73) (Fig. 3) or cardiac heterotaxy (44) (not shown).

\section{Umbilical Arteries}

Ordinarily, there are two umbilical arteries, but in approximately $1 \%$ of births one of these vessels is absent, either because only one developed or because one regressed. It is well known that single umbilical artery is associated with an increased likelihood of other anomalies, particularly of the genitourinary and cardiovascular systems. The entire University of
Michigan Teratology Unit data set $(4,347)$ was surveyed for cases of single umbilical artery: in 112 the left artery was absent, in 126 the right artery was absent. There were eight instances in which the vessel to the placenta was of vitelline origin and eight in which the side of the vessel could not be determined (acardiac fetuses).

Abuhamad et al. [1994] reported a series of 60 cases of single umbilical artery in which absence of the left artery was more commonly associated with anomalies, particularly multiple and cytogenetic anomalies. In my data set, the mean number of different organs or body regions affected did not differ, depending on which artery was absent (Table II). When grouped by systems with malformations, there was only one statistically significant correlation with the side of the arterial 
TABLE II. Correlations of Single Umbilical Artery by Side of Missing Artery

\begin{tabular}{lcc}
\hline $\begin{array}{c}\text { Number of systems with } \\
\text { malformation }\end{array}$ & $\begin{array}{c}\text { Left artery } \\
\text { absent }\end{array}$ & $\begin{array}{c}\text { Right artery } \\
\text { absent }\end{array}$ \\
\hline None & 16 & 11 \\
1 & 14 & 13 \\
2 & 12 & 13 \\
3 & 16 & 17 \\
4 & 19 & 14 \\
5 & 13 & 15 \\
6 & 5 & 5 \\
7 & 1 & 12 \\
8 & 12 & 8 \\
9 & 5 & 3 \\
$10+$ & 3 & 1 \\
Mean & 4.01 & 3.89 \\
\hline
\end{tabular}

System affected

\begin{tabular}{lrr}
\hline Body wall* $^{*}$ & 28 & 20 \\
Central nervous & 17 & 27 \\
Cardiovascular & 66 & 63 \\
Diaphragm & 13 & 8 \\
Gastrointestinal & 50 & 49 \\
Genital** & 60 & 40 \\
Urinary & 52 & 52 \\
Axial skeletal & 33 & 34 \\
Limb & 30 & 29 \\
Respiratory & 16 & 10 \\
Situs ambiguity & 5 & 4 \\
\hline
\end{tabular}

Cytogenetic anomaly

\begin{tabular}{lrr}
\hline Trisomy $13^{*}$ & 11 & 5 \\
Trisomy 18 & 7 & 12 \\
Trisomy $21^{*}$ & 14 & 7 \\
$45, X^{*}$ & 14 & 7 \\
Triploidy** & 1 & 9 \\
\hline
\end{tabular}

$* P<0.10$ (chi $=$ square).

$* * P<0.05(\mathrm{chi}=$ square $)$

absence. Those fetuses with genital anomalies were more likely to be missing the left artery (Table II). In fetuses that had both a single umbilical artery and a cytogenetic anomaly, the missing artery appeared to be more commonly on one side, but the side varied by the karyotype (Table II).

The association between renal malformation and single umbilical artery (89) was examined in more detail. When both kidneys were abnormal, the right artery was absent in 21 cases and the left artery was absent in 33 cases. When only one kidney was abnormal, there was discordance (23) more often than concordance (12) for the side of arterial absence, although this did not reach statistical significance $(P$ 0.063) (Table III). The search for association of single umbilical artery with deviation in the left-right weights of the paired viscera showed a sizable number of outliers, both high and low, reflecting the severity of the associated malformations, with perhaps an excess of high lung outliers (i.e., left lung heavier or right lung lighter than usual) associated with absent left umbilical artery (data not shown).

Blackburn and colleagues [1993a,b] reported that absence of an umbilical artery was associated with a longer foot length on the ipsilateral side. Although Blackburn and I use different methods to measure foot
TABLE III. Renal-arterial concordance

\begin{tabular}{lcc}
\hline Kidney & Umbilical artery & Number \\
\hline Both abnormal & R absent & 21 \\
Both abnormal & L absent & 33 \\
R abnormal & R absent & 6 \\
R abnormal & L absent & 12 \\
L abnormal & L absent & 6 \\
L abnormal & R absent & 11 \\
Concordant & & 12 \\
Discordant & & 23 \\
\hline
\end{tabular}

length, I would expect his reported mean difference in foot length of $2.5 \mathrm{~mm}$ to be apparent with my methodology, but it was not. The vast majority of cases with single umbilical artery showed no appreciable difference (i.e., $<1 \mathrm{~mm}$ ) in foot length. Of 11 cases with a difference in foot length greater than $1 \mathrm{~mm}, 7$ followed Blackburn's prediction, and 4 did not. This offers only weak support for a correlation between umbilical artery absence and accelerated extremity growth.

\section{DISCUSSION}

If in utero growth disturbance affected the left and right sides equally, the usual weight differential between the left and right sides of the paired viscera would be maintained. From the data presented in this article, it can be seen that this is not always the case, and some of the deviations from proportionate growth appear to be correlated with particular malformations or syndromes. The reason for this imbalance in growth is not always evident and merits further consideration. In some cases, such as restriction of lung growth accompanying diaphragmatic hernia or cardiac enlargement, a simple mechanical effect on organ growth can be surmised. It is inferred that the reason the left lung normally weighs less than the right is that the leftpositioned heart impedes left lung growth.

An increased weight of a unilaterally duplicated kidney is to be expected. What is more puzzling is the increased weight of a solitary kidney. Hartshorne et al. [1991] reported that in isolated unilateral renal agenesis, the weight of the remaining kidney was increased, so that the renal weight approximated $80 \%$ of the anticipated weight of two kidneys. Some of the cases in that report were drawn from this data set. Why compensatory overgrowth of the solitary kidney occurs in utero when the fetus presumably depends largely on the placenta for excretory function remains a mystery.

The reversal of the usual weight difference between the adrenal glands in association with hepatic situs inversus may be interpreted as a direct manifestation of the left-right inversion process itself, or alternatively it may be consequent to inversion of the regional vasculature. The fact is that we have no understanding of why the right adrenal normally weighs less than the left.

In other instances, such as the greater equality of adrenal weights and greater restriction of left lung growth in Down syndrome, the basis for the growth aberration is not apparent. Some of the disorders 
examined here, notably trisomies 18 and 13, single umbilical artery, and dyshydramnios, are characterized by excesses of both high and low z-scores, reflecting the complex and variable nature of the associated malformations. This is of some significance because fetuses with cytogenetic anomalies have been described as showing "symmetrical" growth restriction [Reece and Hagay, 1992], whereas a more detailed look at their growth patterns shows a great deal of asymmetry [Barr, 1994].

Over the years, there have been many articles detailing the incidence and morphologic associations of single umbilical artery (not cited here). Few studies have looked at the correlation of side of involvement with the type or laterality of associated malformations. Despite one report to the contrary [Abuhamad et al., 1994], there is no convincing evidence of correlation between the side of arterial absence and the nature or complexity of concomitant malformation. Two exceptions to this are suggested. One is the apparent discordance between the side of the missing artery and unilateral renal abnormality. The other is the preferential absence of right or left artery depending on the karyotype. Study of more cases should verify or refute these observations.

\section{REFERENCES}

Abuhamad A, Shaffer W, Mari G, Copel J, Hobbins J, Evans A. 1994. Single umbilical artery: does it matter which artery is missing? Am J Obstet Gynecol 170:266.

Barr M. 1994. Growth profiles of human autosomal trisomies at midgestation. Teratology 50:395-398.

Barr M. 1997. Growth as a manifestation of teratogenesis: lessons from human fetal pathology. Reprod Toxicol 11:583-587.

Barr M. 1998. Correlates of prenatal visceromegaly. Am J Med Genet 79:249-252.

Barr M, Blackburn WR, Cooley NR. 1994. Human fetal somatic and visceral morphometrics. Teratology 49:487-496.

Blackburn WR, Cooley NR, Stevenson RE. 1993a. New perspectives regarding umbilical cords containing a single artery. Proc Greenwood Genetics Center 12:134

Blackburn W, Cooley NR, Stevenson RE, Martin RA, Jones KL. 1993b. Studies supporting the concept of vascular steal and asymmetric lower extremity growth in the human fetus. Proc Greenwood Genet Center 12:44-46.

Hartshorne N, Shepard T, Barr M. 1991. Compensatory renal growth in human fetuses with unilateral renal agenesis. Teratology 44:710

Pridjian G, Nugent CE, Barr M. 1991. Twin gestation: influence of placentation on fetal growth. Am J Obstet Gynecol 165:13941401

Reece EA, Hagay Z. 1992. Prenatal diagnosis of deviant fetal growth. In: Reece EA, Hobbins JC, Mahoney MJ, Petrie RH, editors. Medicine of the fetus and mother. Philadelphia: JB Lippincott, p 671-685. 